Familles en révolution (1775-1825). Recherches sur les comportements familiaux des populations rurales d'île-de-France, de l'Ancien Régime à la Restauration

Philippe Daumas

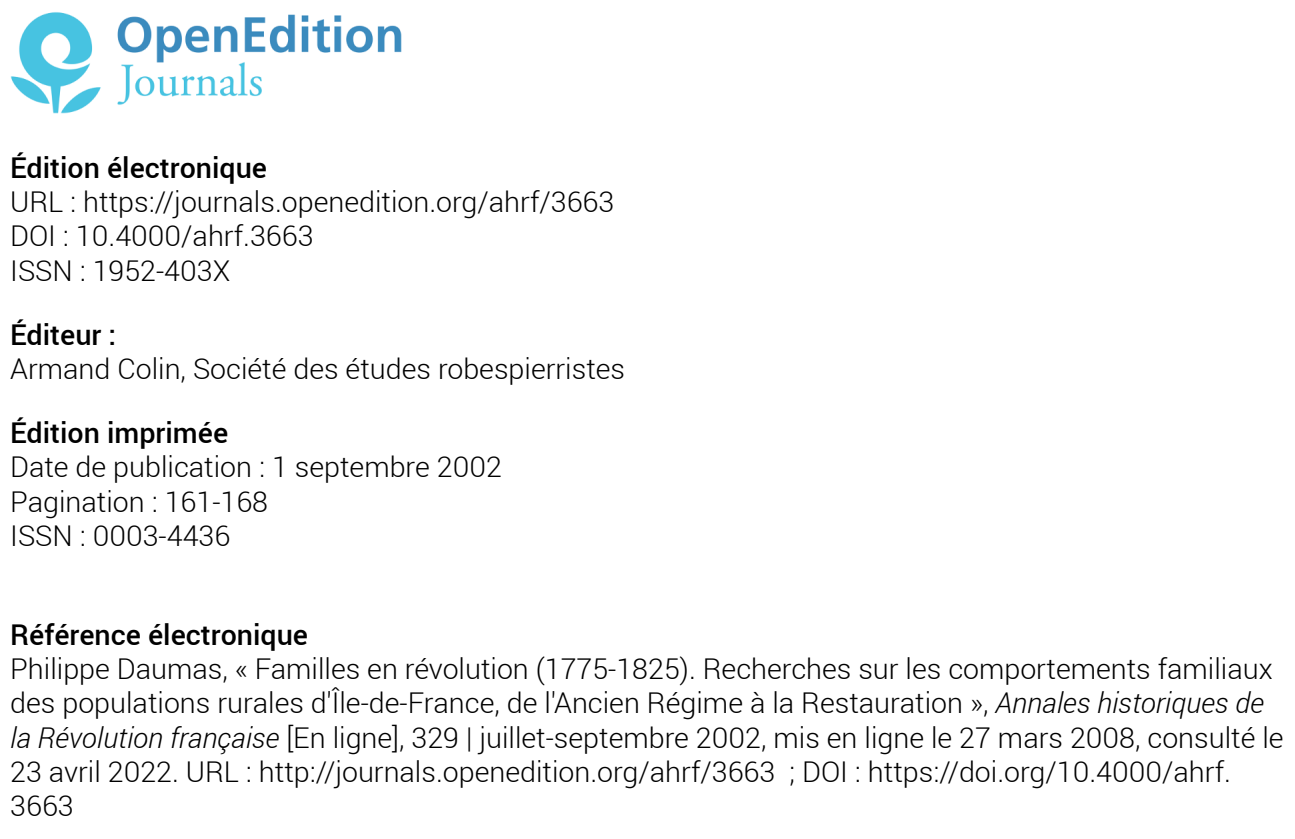




\title{
SOUTENANCE DE THÈSE
}

\author{
FAMILLES EN RÉVOLUTION (1775-1825) \\ RECHERCHES SUR LES COMPORTEMENTS FAMILIAUX \\ DES POPULATIONS RURALES D'ÎLE-DE-FRANCE, \\ DE L'ANCIEN RÉGIME À LA RESTAURATION
}

\author{
PHILIPPE DAUMAS
}

L'effort législatif sans précédent accompli pendant la Révolution française n'avait pas pour unique objectif de faire disparaître l'organisation politique et sociale de l'Ancien Régime. Il exprimait également la volonté de changer radicalement les règles de la vie sociale, les habitudes séculaires, les mentalités, et d'éduquer le peuple par l'exercice d'une citoyenneté active. Lidée essentielle de ce projet politique audacieux, clairement exprimée à travers le concept de «régénération ", est celle d'une nécessité absolue de changer l'homme lui-même pour mener à son terme le progrès social et le rendre irréversible. Mais suffisait-il de promulguer des lois pour atteindre cet objectif? Plus encore qu'à la Contre-Révolution ou à toute forme de résistance consciente et volontaire, cet effort se heurtait nécessairement à de terribles pesanteurs culturelles : le poids des traditions, la force des habitudes, l'immobilisme des mentalités. L'échec de cette entreprise d'acculturation ne se discute pas : on ne change pas les hommes en dix ans. Mais l'importance de l'œuvre législative accomplie et la force de l'événement politique incitent à en mesurer les conséquences : comment les dix années de la Révolution ont-elles influé sur le mode de vie, les comportements, les représentations, les idées de celles et ceux qui les ont vécues? Qu'en est-il resté une fois la Révolution interrompue? Comment l'héritage s'en est-il transmis, sous quelles formes et 
avec quelle ampleur? La réponse à de telles questions exige une observation «sur le terrain » des rapports entre changement et continuité, innovation et tradition, ou pour reprendre la formule de Michel Vovelle, des « jeux de la longue durée séculaire et du temps court d'une Révolution » (1).

Le thème de la famille est sans doute l'un des mieux adaptés à cette tentative de bilan. Cellule de base de la société, sous l'Ancien Régime comme pendant et après une Révolution qui ne l'a jamais remise en cause, la famille est également le cadre de vie où les comportements des individus peuvent être le mieux observés, à partir de multiples sources. Son importance dans l'organisation sociale en a fait de tous temps un enjeu politique majeur. Objet pendant la Révolution d'innombrables déclarations, de débats passionnés et d'un effort législatif sans précédent dans l'Histoire, elle est également un thème récurrent du discours contre-révolutionnaire, dès 1792 et jusqu'à nos jours : de Louis de Bonald à Pierre Gaxotte, en passant par Joseph de Maistre, Taine ou Banville, tous les pourfendeurs de la Révolution lui ont reproché d'avoir «assassiné la famille ». Limportance de l'enjeu fonde ainsi l'intérêt de la recherche : comment apprécier la portée d'un changement politique sans savoir ce qu'il en est advenu dans les faits, si les lois promulguées ont bien été appliquées, et dans quelle mesure elles ont réellement transformé la société ?

Limmensité du sujet, impossible à traiter de manière exhaustive dans le cadre d'une simple thèse, impose des choix d'objectifs et de méthodes nécessairement restrictifs. Le cadre chronologique étendu (de 1775 à 1825), entourant largement la période révolutionnaire, implique en contrepartie un cadre géographique plus limité : onze villages d'île-de-France (2), représentant une population d'environ 6000 habitants, dans un milieu rural fortement marqué par la proximité de la capitale. Les sources utilisées privilégient la proximité du terrain et la rencontre avec les milieux populaires. Le dépouillement systématique des registres paroissiaux, d'état civil et de catholicité des onze villages fournit un corpus de naissances et de mariages (3) suffisant à l'observation d'indicateurs aussi variés que le respect des sacrements et des prescriptions religieuses, le choix du conjoint, la sexualité hors mariage ou l'évolution du système de prénomination. La recherche de formes d'expression plus directe d'idées ou de sentiments a été conduite principalement dans les archives judiciaires, une source d'une extrême richesse et encore largement inexploitée. Leurs actes livrent souvent la description détaillée de situations vécues ou de comportements, la transcription de déclarations orales, le détail des argumentaires, autant d'instruments

(1) M. VOVELLE, La mentalité révolutionnaire, Paris, 1985, p. 267.

(2) Aulnay, Chennevières, Gennevilliers, Ivry, Noiseau, Orly, Ormesson, Saint-Rémy-lès-Chevreuse, Sevran, Thiais et Trappes.

(3) L'enquête porte sur 2895 mariages, 10863 naissances et l'attribution de 22227 prénoms, entre 1775 et 1825 . 
pour percer, comme le suggère Michel Vovelle, "le mur de silence de ceux qui n'ont pas écrit » (4). Ainsi, les actes de huit justices seigneuriales (5) et de cinq justices de paix (6) ont permis d'étudier 374 «affaires de famille», riches de formes d'expression plus directement lisibles que les données chiffrées. Paradoxalement, les archives notariales répondent moins à cette attente : bien que de nombreux actes y concernent la vie familiale, leur rédaction très stéréotypée ne permet guère l'expression personnelle des individus. Elles ne sont donc utilisées ici que très ponctuellement.

Le choix des sources, comme celui des thèmes abordés, s'avère extrêmement frustrant. Retenir les indicateurs les plus pertinents par leur caractère démonstratif, la fiabilité des sources ou l'adéquation aux objectifs de la recherche, implique le renoncement à d'autres pistes tout aussi passionnantes, comme par exemple les réseaux de relations, la gestion et la transmission des patrimoines, les transformations du cadre de vie, l'éducation et la place des enfants au sein de la famille, les attitudes face à la vieillesse et à la mort. Certains de ces thèmes ont déjà fait l'objet de remarquables travaux, mais concernant principalement l'Ancien Régime. Pour la période révolutionnaire, ces chantiers demeurent encore largement ouverts.

L'idée directrice de cette étude est de tenter la synthèse d'indicateurs multiples dans une «lecture culturelle" permettant de mettre en évidence des évolutions significatives et d'apprécier dans quelle mesure elles sont déterminées par la Révolution. L'approche quantitative, alimentée par les statistiques, est toujours complétée par une approche qualitative, à partir d'exemples, de témoignages ou de situations vécues, ce qui répond non seulement à la nécessité de croiser les sources et de diversifier les approches, mais aussi à la volonté d'écrire une Histoire «vivante». Sur chaque thème et chaque indicateur, la réflexion méthodologique a été poussée le plus loin possible (fiabilité des sources, multiplication des questionnements et des hypothèses, différentes lectures possibles, croisement des informations, nécessité de nuancer les conclusions et de remettre en cause les idées reçues), non avec la prétention de fixer définitivement des règles, mais plus modestement d'apporter une contribution personnelle à une réflexion collective qui se construit dans le cours même de la recherche, par la confrontation des expériences et l'échange des idées.

Le milieu rural d'île-de-France présente des caractères profondément originaux. Bien avant la Révolution, il diffère totalement de l'image traditionnelle de villages «immobiles» ou repliés sur eux-mêmes. La proximité

(4) M. VOVELLE, op. cit., p. 15.

(5) Bailliage d'Ivry (1775-1790), bailliage de Pantin (1775-1790), bailliage du marquisat d'Ormesson (1775-1790), bailliage royal de Vincennes (1775-1791), prévôté de Gennevilliers (1775-1790), prévôté d'Orly (1775-1790), prévôté de Sucy (1775-1790), prévôté de Thiais, Choisy et Grignon (1775-1789).

(6) Justices de Paix de Colombes (1791-an X), Pantin (1791-1825), Sucy (1791-an VIII), Villejuif (1791-1825) et Vincennes (1791-1825). 
de Paris et l'importance des voies de communication y favorisent des formes variées de relations avec la capitale : échanges commerciaux, changements de résidences, mise en nourrice d'enfants parisiens, réseaux de relations familiales, une constante circulation des hommes et des biens qui favorise celle des informations et des idées. L'importance des conflits d'intérêts, comme la diversité des attitudes politiques, morales ou religieuses, y marquent fortement les limites de l'esprit communautaire et de la cohésion sociale. Ces conditions particulières, de même qu'un contexte marqué par les affrontements politiques et idéologiques, les crises économiques et les campagnes militaires, confèrent à ce milieu rural relativement atypique une réceptivité particulière aux changements culturels. Mais la diversité des villages étudiés (taille, population, activités, proximité de Paris), si elle permet de déceler les retombées du contexte local sur les pratiques culturelles, ne saurait en aucun cas en faire un "échantillon représentatif " des campagnes d'île-de-France, encore moins de la France entière.

Dans ce contexte pourtant favorable, les lois révolutionnaires connaissent des fortunes diverses. La laïcisation de l'état civil, réalisée sans difficultés, apparaît comme le point de départ de tout le dispositif de régénération de la famille : en privant l'Église de son principal moyen de contrôle sur la vie privée, elle ouvre aux citoyens un espace de liberté indispensable à l'accomplissement du changement. De même, l'abaissement de l'âge de la majorité, les lois successorales ou la mise en place d'institutions judiciaires facilitant le règlement amiable des conflits familiaux, s'appliquent sans difficultés et constituent donc les principaux succès de la législation révolutionnaire. Cependant, les lois les plus innovantes, celles qui remettent le plus fortement en cause la structure familiale traditionnelle, connaissent un échec indiscutable. C'est le cas en particulier du divorce, beaucoup plus rare dans les campagnes d'Île-de-France que dans la capitale elle-même, ou encore de l'adoption, dont on ne trouve aucune trace dans les villages étudiés. Sous le Consulat, puis l'Empire et même la Restauration, la législation révolutionnaire n'est pas totalement abolie. Elle est profondément remaniée, dans le sens de la restauration de l'autorité paternelle et maritale, mais sans jamais aller jusqu'au rétablissement complet de l'ordre ancien. $\mathrm{La}$ portée du changement révolutionnaire est ainsi indirectement mise en évidence : s'il n'est pas totalement réversible dans les lois, c'est bien parce qu'il demeure solidement ancré dans les esprits.

Son effet le plus profond et le plus durable est sans aucun doute le relâchement de l'emprise religieuse sur la vie des individus et des familles. Si la laïcisation de l'état civil, la constitution civile du clergé, les campagnes de déchristianisation et pour finir l'interdiction du culte ne sont pas venues à bout de l'influence de la religion, leurs conséquences sont cependant indéniables : au rétablissement du culte en l'an III, l'Église paraît considérablement affaiblie et se voit contrainte à un travail de patiente reconquête 
vis-à-vis de paroissiens qui ne lui sont plus acquis $a$ priori. Les sacrements, en particulier le baptême, retrouvent en grande partie leur importance après le rétablissement du culte. Mais les simples prescriptions, jugées aliénantes pour la liberté individuelle et non indispensables au salut, ne résistent pas aux nouvelles aspirations. Ainsi, l'élévation constante de l'âge au baptême témoigne sans doute d'un souci accru de la santé de l'enfant, affirmation implicite de l'importance de la vie terrestre par rapport au salut de l'âme. De même, le détachement vis-à-vis des temps clos et la multiplication des dispenses pour les mariages montrent que les conditions matérielles ou les convenances personnelles l'emportent de plus en plus souvent sur les exigences de l'Église. Plus nettement encore, la multiplication des prénoms librement choisis hors du martyrologe ou des modes de transmission traditionnels affirme la liberté de l'individu face aux habitudes sociales et aux contraintes religieuses. De manière plus diffuse mais non moins réelle, la progression des idées nouvelles remet en cause la morale chrétienne : les amours plus libres, le couple plus égalitaire, l'expression de l'individualisme dans les comportements, concrétisent la perte d'influence de la religion.

Peu à peu, l'Église semble accepter en grande partie ces évolutions. Face à la pression de paroissiens animés de sentiments nouveaux et d'une conception de la foi plus intériorisée, moins ritualisée, les autorités religieuses semblent n'avoir d'autre alternative que de s'adapter ou de perdre leur influence. Il semble que l'Église ait considéré comme prioritaire le maintien des sacrements et la reprise du catéchisme, essentiels pour la préservation de l'avenir, acceptant en échange de faire d'importantes concessions dans le domaine des simples prescriptions. Même si ce n'est pas la foi ellemême qui est remise en question mais sa ritualisation et les contraintes qu'elle impose, ce relâchement de l'emprise religieuse sur la morale et les comportements individuels est sans doute un des effets les plus marquants de la période révolutionnaire.

Plus encore que l'influence de la religion ou de la Contre-Révolution, la volonté de changement social et culturel devait surmonter l'inertie des mentalités. Les « attitudes révolutionnaires » peuvent donc être définies par leur degré de rupture avec les mœurs et les idées de l'Ancien Régime, le mot «rupture » étant pris ici dans son sens le plus large : il ne s'agit pas seulement de mesurer le degré d'application des lois nouvelles, mais d'apprécier de quelle manière les individus s'approprient l'espace de liberté qui leur est ouvert pour penser et vivre autrement. Si de nombreux indicateurs témoignent d'un «vent de liberté » touchant tous les aspects de la vie familiale, celui-ci se manifeste moins par l'invention de comportements nouveaux que par l'amplification de phénomènes déjà observés sous l'Ancien Régime, mais de manière très minoritaire et marginale, presque toujours privilège des classes socialement et culturellement favorisées. La Révolution les multiplie, les banalise et les démocratise. Dans le même temps, ces «prises de liberté " 
témoignent de l'évolution des rapports sociaux et de l'émergence de nouvelles valeurs morales, qu'elles soient proclamées ou implicites.

C'est tout d'abord la montée de l'individualisme, souvent présenté comme une dérive de l'exercice de la liberté individuelle, dont il est effectivement indissociable, mais qui ne s'oppose pas de manière systématique à la contrainte collective. Il s'exprime souvent dans les conflits, lorsque l'intérêt personnel l'emporte sur la force du lien familial. De manière plus diffuse, il s'exprime à travers des décisions personnelles en rupture avec les habitudes anciennes. Le choix des prénoms en offre l'exemple le plus significatif : l'accroissement considérable du corpus, les nombreuses créations de prénoms, l'éclatement de l'ancien système de références, la dissociation accrue des prénoms masculins et féminins, toutes ces évolutions montrent que la logique de l'individualisation s'impose de plus en plus face à celle de l'identification au groupe familial. L'individualisme s'exprime enfin, de manière implicite et souvent inconsciente, à travers toutes les formes du refus des contraintes collectives. Amours libres, concubinage, rejet de la religion sous toutes ses formes, concrétisent la recherche d'un bonheur ou d'un équilibre strictement personnels, conçus indépendamment du lien social.

L'importance accordée aux sentiments et à leur expression, de plus en plus libre et de plus en plus forte, apparaît également comme un effet de la période. Si le sentiment prédomine dans toutes les formes d'amour qui précèdent le mariage ou qui s'en dispensent, en particulier le concubinage, il apparaît aussi indirectement dans ces unions où ni l'âge des conjoints, ni leur condition sociale ne correspondent aux normes traditionnelles. Écarts d'âges ou de fortune, mariages de filles enceintes ou de filles mères, mariages entre conjoints jeunes, conclus à la hâte et parfois contre l'avis des parents, sont de plus en plus fréquents et semblent devoir plus aux sentiments qu'à l'argent ou à la nécessité de «s'installer ». Les archives judiciaires témoignent des difficultés de jeunes gens à imposer à leurs parents des mariages d'amour, mais aussi de ruptures de promesses de mariage faute de sentiments. Le couple marié ne subit-il pas lui aussi cette influence? Pendant la Révolution, le décalage entre nuptialité et fécondité, considéré par les démographes comme l'effet d'un comportement «malthusien », peut également indiquer que le couple n'est pas seulement voué à la reproduction mais qu'il devient de plus en plus une fin en lui-même, les sentiments s'imposant face aux nécessités de la vie. La nouveauté ne réside pas bien sûr dans l'existence des sentiments, mais plutôt dans leur extériorisation accrue, leur influence plus forte sur les actes et la détermination des choix, enfin et surtout l'extension de ces attitudes à toutes les classes sociales. Dans ce domaine comme dans bien d'autres, la Révolution annonce les sensibilités du XIXe siècle.

Les bases sociologiques du changement sont assez difficiles à cerner. Il semble impossible de définir objectivement des «milieux révolutionnaires » ou des « milieux conservateurs », tant les variations observées paraissent peu 
significatives. Pour pratiquement tous les indicateurs, la répartition sociale est très proche de celle de l'ensemble de la population. On peut observer cependant que le milieu ouvrier, catégorie naissante et concentrée dans certaines communes, paraît moins soumis à la religion et à la tradition que les autres classes sociales, donc plus sensible aux attitudes novatrices ou en rupture avec les normes. Malgré quelques modestes écarts sur certains indicateurs, les ruraux participent eux aussi à toutes les formes d'innovation et de libéralisation des mœurs, ce qui ne correspond pas à l'image traditionnelle d'un milieu paysan conservateur, borné ou replié sur lui-même. On peut enfin s'étonner que le personnel révolutionnaire n'apparaisse nulle part et à aucun moment comme l'élément moteur du changement... En fait, on ne peut opposer de manière manichéenne et réductrice les forces du progrès à celles de l'immobilisme, des groupes sociaux imaginatifs et innovants à d'autres plus conservateurs, des attitudes nouvelles à des traditions. Les pratiques culturelles ne s'analysent pas d'une manière aussi tranchée, aussi définitive, simplement parce que leurs auteurs ne les vivent pas et ne les perçoivent pas de cette manière. Même si les événements politiques de la période suggèrent l'idée d'une bipolarisation idéologique, le rapport entre changement et continuité, tradition et innovation, paraît beaucoup plus complexe : ils peuvent coexister ou s'affronter au gré des circonstances et d'une manière pas toujours cohérente, au niveau d'un groupe social comme à celui d'une famille ou même d'un individu. Le changement culturel ne se réalise ni en un jour, ni en bloc, mais selon une grande diversité de processus, pouvant comporter des contradictions, des décalages; des hésitations, ou même des retours en arrière, et parfois de curieuses «synthèses » entre des idéologies pourtant parfaitement antagonistes. Si ces attitudes peuvent nous surprendre, n'oublions pas leur caractère fugitif, saisi au hasard des actes, simples fragments de vies dont le déroulement entier nous échappe. De plus, elles sont par définition temporaires, car les pratiques culturelles, à l'image de la pensée, ne sont ni statiques, ni achevées : entre l'héritage du passé et la construction du futur, elles sont toujours en devenir.

Réalité bien vivante, le changement révolutionnaire marque fortement les comportements, même s'il demeure à certains égards fragile, complexe et inégal. Dans ce produit de la rencontre entre «temps court» et «temps long", on retrouve aussi bien l'influence de lentes évolutions amorcées au cours du XVIIIe siècle, que les effets brutaux et immédiats de l'événement révolutionnaire. Dans une ambiance culturelle marquée par l'affaiblissement de la religion et l'exigence du changement social, sous l'effet d'une idéologie prônant la liberté et l'égalité, la Révolution entraîne une accélération et une amplification des évolutions en cours. Elle y ajoute les effets d'une volonté de régénération concrétisée par des lois nouvelles, des décisions politiques et une pression idéologique permanente. Après le 18 Brumaire, les changements politiques ont d'importantes répercussions sur la législation, mais ne semblent 
finalement affecter les comportements familiaux que de manière très modeste et temporaire. La période 1800-1810 marque un fléchissement ou un léger recul des évolutions amorcées pendant la Révolution, perceptible pour de nombreux indicateurs. Mais elle est suivie, à partir de 1810-1815, d'une nouvelle et importante période de changements : dans tous les domaines, les évolutions reprennent ou s'accélèrent, comme si subitement les traditions devenaient moins pesantes, la religion moins influente, les esprits plus libres. Si on n'assiste pas à un bouleversement total des conceptions de la famille, d'ailleurs impensable dans un climat aussi conservateur et répressif que celui de l'Empire et de la Restauration, cette "deuxième vague " de changements culturels n'en est pas moins profonde et durable, puisqu'elle se prolonge de manière continue jusqu'à la fin de la période étudiée. Elle paraît plus significative encore que celle des années 1793-1794, dans la mesure où elle est moins directement liée à la conjoncture politique. En l'an II, les changements culturels semblent avoir résulté avant tout de la conjonction des événements et de l'action du pouvoir révolutionnaire. Quinze ou vingt ans plus tard, cette pression de l'événement ou du climat politique n'existe plus. Bien au contraire, le contexte des années 1810-1825 parait nettement défavorable au changement culturel. Celui-ci semble donc plus fortement ancré dans les mentalités, et par conséquent plus significatif, mais l'explication en est plus incertaine.

La chronologie des faits suggère fortement une hypothèse : les acteurs de ces nouveaux changements sont les enfants des révolutionnaires de l'an II. Nés pendant la Révolution ou peu avant, ils ont été élevés dans un tout autre contexte que leurs parents, avec d'autres valeurs morales, d'autres références, d'autres modèles, et sans doute un esprit plus libre. Cette «deuxième révolution culturelle » constitue en quelque sorte le prolongement de celle de l'an II. Si la Révolution a été interrompue avant d'avoir pu forger un «homme nouveau ", si ses acteurs, trop marqués sans doute par leur éducation et leurs habitudes de vie, n'ont pu accomplir eux-mêmes des mutations culturelles à la fois profondes et durables, ils ont néanmoins créé les conditions des changements ultérieurs en élevant leurs enfants sur la base d'idées nouvelles et d'une plus grande liberté d'esprit. Cette hypothèse ne saurait en aucun cas exprimer une conception cyclique de l'Histoire. Elle rappelle simplement, s'il en était besoin, le rôle de l'éducation dans la réalisation et le prolongement du changement culturel. C'est de cette manière qu'un événement bref, mais d'une portée exceptionnelle, peut inscrire ses effets dans la longue durée.

Philippe Daumas

Université de Rennes 2

16 boulevard Pasteur

75015 Paris

daumas.martin@wanadoo.fr 